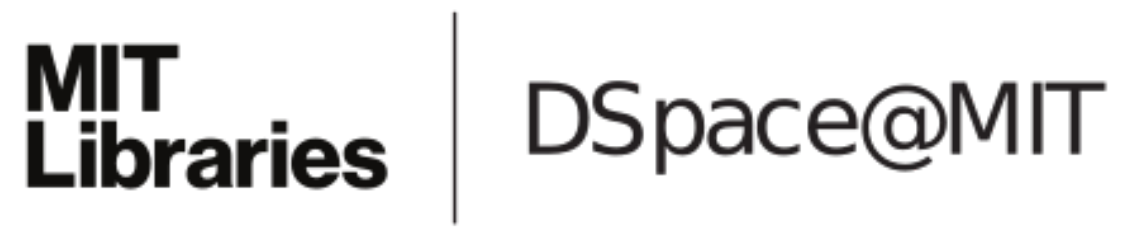

\author{
MIT Open Access Articles
}

Thermal ghost imaging with averaged speckle patterns

The MIT Faculty has made this article openly available. Please share how this access benefits you. Your story matters.

Citation: Zerom, Petros et al. “Thermal Ghost Imaging with Averaged Speckle Patterns."

Physical Review A 86.6 (2012). (C) 2012 American Physical Society

As Published: http://dx.doi.org/10.1103/PhysRevA.86.063817

Publisher: American Physical Society

Persistent URL: http://hdl.handle.net/1721.1/76801

Version: Final published version: final published article, as it appeared in a journal, conference proceedings, or other formally published context

Terms of Use: Article is made available in accordance with the publisher's policy and may be subject to US copyright law. Please refer to the publisher's site for terms of use. 


\title{
Thermal ghost imaging with averaged speckle patterns
}

\author{
Petros Zerom, ${ }^{1, *}$ Zhimin Shi, ${ }^{1,}, \dagger$ Malcolm N. O'Sullivan, ${ }^{1}$ Kam Wai Clifford Chan, ${ }^{2}$ Molly Krogstad, ${ }^{1,3}$ \\ Jeffrey H. Shapiro, ${ }^{4}$ and Robert W. Boyd ${ }^{1,5}$ \\ ${ }^{1}$ The Institute of Optics, University of Rochester, Rochester, New York 14627, USA \\ ${ }^{2}$ Rochester Optical Manufacturing Company, 1260 Lyell Avenue, Rochester, New York 14606, USA \\ ${ }^{3}$ Department of Electrical, Computer, and Energy Engineering, University of Colorado at Boulder, Boulder, Colorado 80309, USA \\ ${ }^{4}$ Research Laboratory of Electronics, Massachusetts Institute of Technology, Cambridge, Massachusetts 02139, USA \\ ${ }^{5}$ Department of Physics, University of Ottawa, Ottawa, Ontario, Canada K1N 6N5
}

(Received 15 June 2012; published 17 December 2012)

\begin{abstract}
We present theoretical and experimental results showing that a thermal ghost imaging system can produce images of high quality even when it uses detectors so slow that they respond only to intensity-averaged (that is, "blurred") speckle patterns, as long as the collected signal variation is predominantly caused by the random fluctuation of the incident speckle field rather than other noise sources. In our experimental study, we show that the quality of the ghost image is not degraded when as many as 25 speckle patterns are averaged together for each measurement. This surprising result comes from the fact that the averaging of speckle patterns leads to a decrease in the contrast but not in the kurtosis, and the image quality of a ghost imaging system is dependent on the kurtosis rather than the contrast ratio of the illuminating field. These results suggest that a broad class of imaging systems based on the use of speckle techniques can be implemented even using detectors that respond slowly on the time scale of the fluctuating speckle pattern.
\end{abstract}

DOI: 10.1103/PhysRevA.86.063817

PACS number(s): 42.30.Ms, 42.30.Va, 42.50.Ar

\section{INTRODUCTION}

The term "speckle pattern" conventionally refers to the intensity distribution produced by the mutual interference of a set of randomly generated wave fronts, such as those obtained when scattering a coherent laser beam off a rough surface or in the propagation of star light through the turbulent atmosphere. In recent years, a diverse range of applications has been developed to make use of the speckle phenomena in the context of astronomy [1], metrology for biomedical applications [2-4], imaging of strongly interacting quantum systems [5], random lasers [6], etc.

The success of many speckle-based techniques relies, more or less, on the facts that a speckle field can have high spatial and temporal randomness and that the detecting devices have enough spatial and temporal resolution to monitor the variations of individual speckles. The contrast ratio, which in many circumstances is used to quantify the amount of variation within a speckle pattern, can be defined as $K=\sigma(I) /\langle I\rangle$, where $\sigma(I) \equiv \sqrt{\left\langle I^{2}\right\rangle-\langle I\rangle^{2}}$ is the standard deviation of the intensity variation of the speckles and $\langle\cdots\rangle$ denotes either temporal or spatial ensemble average.

When the detector used in such a system is slow in the sense that its refresh rate cannot keep up with the temporal variations of the illuminating speckle field, the effective illuminating field that the system measures essentially becomes the intensity average (or sum) of multiple mutually uncorrelated speckle patterns. In such cases, the contrast ratio $K$ of such an intensity-averaged speckle pattern scales with the speckle averaging factor $M$ as $M^{-1 / 2}$ [7] [cf. Eq. (9) and Fig. 2]. The quantity $M$ is calculated as $\tau_{\mathrm{d}} / \tau_{\mathrm{c}}$, that is, as the ratio between the integration time $\tau_{\mathrm{d}}$ of the detector and the coherence time

\footnotetext{
${ }^{*}$ P. Zerom and Z. Shi contributed equally to this work.
}

${ }^{\dagger}$ zshi@optics.rochester.edu $\tau_{\mathrm{c}}$ of the random speckle field. In other words, $M$ indicates the number of independent speckle patterns that are averaged together in each measurement. As a consequence of the reduced contrast ratio, the performance of many speckle-based metrology and imaging techniques would quickly deteriorate as the response (integration) time of the detector increases [4], i.e., as $M$ increases.

Recently, speckle fields have been utilized to perform ghost imaging [8-17], an indirect imaging method that acquires the image of an object through spatial intensity correlation measurements. Compared to conventional imaging techniques, ghost imaging uses a nonspatially resolving bucket detector to collect the optical signal directly from the object either through reflection or transmission, and therefore it can be advantageous in scenarios where using a detector array is restricted or difficult.

One might naturally expect that a ghost imaging system that uses slow detectors would produce images of degraded quality, as is the case in many other speckle-based imaging methods. However, in this article we show both theoretically and experimentally that this is actually not the case and that the image quality of a thermal ghost imaging system can remain high even though the refresh rate of the detectors is much slower than the coherence time of the illuminating speckle field, as long as the fluctuations in the detected signal are due predominantly to the randomness of the speckle pattern itself and not due to noise in the detection system. Similar theoretical results were presented earlier in Ref. [17]; the present paper provides an experimental demonstration of this result.

Before proceeding with our analysis, a few preliminary comments are in order. For years there has been some controversy in the literature regarding the origin of pseudothermal ghost imaging (cf. Refs. $[18,19]$ ). The key issue is whether it can be explained as arising from classical intensity-fluctuation correlations between speckle patterns illuminating the object and the reference detector or whether it can only be understood 


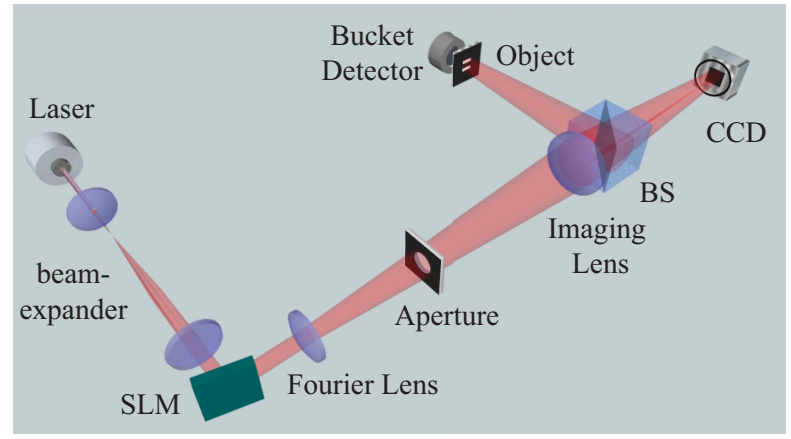

FIG. 1. (Color online) Schematics of our thermal ghost imaging setup. The spatial light modulator (SLM) is used to impress a sequence of speckle patterns onto the laser field. BS: beam splitter; CCD: imaging detector.

as coming from nonlocal two-photon interference. It has recently been shown that it is possible to unify these two disparate interpretations [20], and in our opinion objections to this unification [21] have been successfully refuted [22]. Thus, in this article we limit our analysis to the treatment of classical fields because such an approach is both convenient and appropriate for the description of the high-intensity (specklelimited) regime in which our experiments are performed. We note further that the good agreement between theory and experiment obtained in this work provides further evidence that a quantum description of the process is not needed.

\section{THEORY}

Figure 1 illustrates our thermal ghost imaging system. A collimated laser beam is used to illuminate a phase-only spatial light modulator (SLM), which is programmed to impose a uniformly distributed random-phase distribution onto the incident beam [23]. The first-order diffracted beam forms speckle patterns with negative exponential intensity distributions [1] at the focal plane of a Fourier lens and is used as the illuminating source. The generated illuminating field is then projected, using an imaging lens and a beam splitter, onto the object and reference planes. In the object plane, the speckle pattern is projected onto a transmissive object, and all the transmitted light is collected by a large-area bucket detector placed behind the object. In the reference arm, the intensity distribution of the illuminating speckle field is directly collected by a detector array, a camera in our case. By using many uncorrelated speckle patterns and correlating the signals collected by the bucket detector and the camera, one can obtain a ghost image of the object using the following background-subtracted correlation function [24]:

$$
G(\vec{x})=\frac{1}{N} \sum_{n=1}^{N} I_{\mathrm{B}}^{(n)} I^{(n)}(\vec{x})-\frac{1}{N^{2}} \sum_{n=1}^{N} I_{\mathrm{B}}^{(n)} \sum_{n=1}^{N} I^{(n)}(\vec{x}),
$$

where

$$
I_{\mathrm{B}}^{(n)}=\sum_{x} I^{(n)}(\vec{x}) O(\vec{x})
$$

is the total signal collected by the bucket detector for the $n$th measurement, $N$ denotes the total number of measurements, $I^{(n)}(\vec{x})$ is the intensity of the illuminating field collected by the camera for the $n$th measurement at location $\vec{x}$, and $O(\vec{x})$ is the transmission function of the object. For simplicity, we here assume that the object has binary transmission; i.e., the value of $O(\vec{x})$ is either zero or unity. Note that the second term in Eq. (1) is the product background from two uncorrelated signals, and by subtracting this term, we ensure that $\langle G(\vec{x})\rangle=0$ for any pixels where $O(\vec{x})=0$.

For an object with binary transmission, the image quality of the obtained "ghost" image can be quantified using the contrast-to-noise ratio (CNR), which can be defined by the following expression [24]:

$$
R_{\mathrm{CN}} \equiv \frac{\left\langle G_{1}\right\rangle-\left\langle G_{0}\right\rangle}{\sqrt{\sigma_{1}^{2}+\sigma_{0}^{2}}}
$$

where $\left\langle G_{1}\right\rangle$ and $\left\langle G_{0}\right\rangle$ are the ensemble average of the ghost image signal at any pixel where the transmission is 1 and 0 , respectively, and $\sigma_{1}^{2}$ and $\sigma_{0}^{2}$ are the corresponding variances, respectively.

One can derive analytically an expression for the CNR of such a ghost imaging system in terms of the statistical properties of the illuminating speckle field with three simplified but reasonable assumptions: (1) the intensity of the illuminating patterns are statistically independent of each other, (2) the intensity at each pixel is independent from that at each other pixel, and (3) the detected signal fluctuation is primarily given by the random intensity variation of the speckle fields, and other noise sources, e.g., detector dark noise, can be neglected. Note that the second assumption implies that we can replace the ensemble averages in Eq. (3) with spatial average, and in fact we use spatial average in our simulation and experiment to calculate the CNR of the obtained ghost image.

Under these three assumptions, one can obtain the following expression (see Appendix for details) for the expected CNR of the ghost image:

$$
R_{\mathrm{CN}}=\left[\frac{N-1}{(2 T-2+3 / N)+(1-1 / N)\left(\gamma_{I} / \sigma_{I}\right)^{4}}\right]^{1 / 2},
$$

where $T$ is the ratio between the total transmitting area of the object and the average speckle size of the illuminating speckle pattern [24,25], $\sigma_{I}^{2} \equiv\left\langle I^{2}\right\rangle-\langle I\rangle^{2}$ and $\gamma_{I}^{4} \equiv\left\langle(I-\langle I\rangle)^{4}\right\rangle$ are the second and fourth moments about the mean, respectively, of the intensity fluctuation for each illuminating speckle field, and we use here the shorthand $I \equiv I(\vec{x})$. Note that Eq. (4) is valid for any illuminating field with arbitrary statistical properties, as long as the pixel intensities are statistically independent. This expression also indicates that the image quality of a thermal ghost imaging system is affected by the fourth standardized moment $\left(\gamma_{I} / \sigma_{I}\right)^{4}$, also known as the kurtosis, of the intensity fluctuation of the illuminating field, rather than by the contrast ratio $K$ of a speckle pattern as in many conventional specklebased methods.

When the detectors are fast enough to record individual speckle patterns, which obey negative exponential intensity statistics [7], one can show that $\gamma_{I}^{4}=9\langle I\rangle^{4}$ and $\sigma_{I}^{2}=\langle I\rangle^{2}$. Consequently, the CNR of the ghost image with background subtraction is given by [25]

$$
R_{\mathrm{CN}}=\left[\frac{N-1}{2 T+7-6 / N}\right]^{1 / 2} .
$$


When the detectors are slow, the ghost imaging system responds only to the intensity average of $M$ independent speckle patterns for each measurement. In such cases, the ghost image can still be expressed using Eq. (1), but with modified expressions for the bucket detector signal and the spatially resolved camera signal to take into account the intensity sum of $M$ independent speckles patterns for each measurement, specifically,

$$
I_{\mathrm{B}, \mathrm{M}}^{(n)}=\sum_{m=1}^{M} I_{\mathrm{B}}^{(n, m)}
$$

and

$$
I_{\mathrm{M}}^{(n)}(\vec{x})=\sum_{m=1}^{M} I^{(n, m)}(\vec{x}) .
$$

Using straightforward mathematics, one can show that the fourth-order moment about the mean $\gamma_{I_{M}}^{4}$ and the variance $\sigma_{I_{M}}^{2}$ of the intensity of the effective illuminating speckle field are given by

$$
\gamma_{I_{M}}^{4}=3(M+2) M\langle I\rangle^{4}=\frac{3(M+2)}{M^{3}}\left\langle I_{M}\right\rangle^{4}
$$

and

$$
\sigma_{I_{M}}^{2}=M\langle I\rangle^{2}=\frac{1}{M}\left\langle I_{M}\right\rangle^{2},
$$

where $\langle I\rangle$ and $\left\langle I_{M}\right\rangle$ are the expected values of the intensity for each independent speckle pattern and the intensity-averaged speckle field at any pixel, respectively. Substituting these relations into Eq. (4), one can obtain the following remarkably simple expression for the CNR of a ghost imaging system that only responds to intensity-averaged speckle fields:

$$
R_{\mathrm{CN}}=\left[\frac{N-1}{2 T+1+6 / M-6 /(M N)}\right]^{1 / 2} .
$$

The above expressions show that, even though the contrast ratio of the effective speckle fields "seen" by a ghost imaging system decreases rapidly as the detectors become slow, the quality of the ghost image actually remains approximately the same as long as the transmitting area of the object is much larger than the spatial coherence area of the individual speckle fields. This surprising result comes from the fact that the quantity that affects the image quality of a ghost imaging system is the kurtosis of the intensity fluctuation of the illuminating speckle field, which actually converges to a constant value of 3 as $M$ becomes larger than 10 (see Fig. 2). Furthermore, in most practical situations, the transmitting area of the object is much larger than the coherence area of individual speckle field, i.e., $T \gg 1$. In such cases, the image quality becomes essentially independent of the speckle averaging factor $M$ [cf. Eqs. (4) and (10)]. Note that our Eq. (4) is a generalized result for thermal ghost imaging systems using illuminating fields having arbitrary statistical properties. Our Eq. (10) is a special case of this result for an illuminating field in the form of the intensity sum of multiple speckle patterns. The result of Eq. (10) is consistent with the earlier prediction given in Table I of Ref. [17].

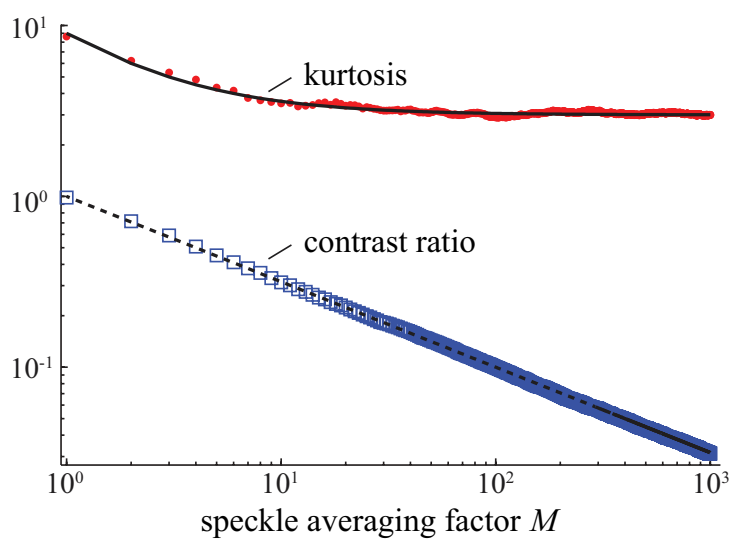

FIG. 2. (Color online) Speckle contrast ratio $K$ and kurtosis $\left(\gamma_{I} / \sigma_{I}\right)^{4}$ as functions of the speckle averaging factor $M$. Here the lines are the theory [cf. Eqs. (8) and (9)], and symbols are the calculated results from one typical numerical simulation realization $[1,23]$.

\section{EXPERIMENTAL RESULTS}

In our experiment, our transmissive object is a double slit, whose transmitting area is approximately 200 times the average speckle size of each independent speckle field, i.e., $T \approx 200$. The CCD and bucket signals are averaged for $M$ uncorrelated speckle patterns before the two signals are correlated using Eq. (1) to mimic the use of slow detectors that respond to the average of $M$ independent speckle patterns. Note that, for $M=1$, our system reduces to a conventional ghost imaging system in which the detectors respond to each independent speckle pattern.

We make the measurements for speckle averaging factor $M$ equal to $1,5,15$, and 25 , respectively, to study quantitatively the effect of the response time of the detecting system on the quality of the "ghost image" that we obtain. For each value of $M$, we take 10000 effective measurements. Figures 3(a) and 3(b) show two typical illumination patterns recorded by the CCD camera for $M=1$ and $M=25$, respectively. The speckle contrast ratio $K$ for the two cases is 1 and 0.2 , respectively. The ghost images after 10000 effective measurements for the two cases are shown in Figs. 3(c) and 3 (d), respectively. One sees that there is no obvious difference in image quality, which is consistent with our theoretical prediction.

To better demonstrate our theoretical predictions, we plot in Fig. 4 (as symbols) our measured CNR of the ghost image as a function of the number of measurement $N$ for four different values of the speckle averaging factor $M$. It can be seen that even though the response time of the detectors in the four cases is very different, there is no obvious difference in the resulting image quality. Also shown in Fig. 4 (as lines) are the results of numerical simulation. The agreement between simulation and laboratory measurement is very good. The slight disagreement may be due to other noise sources (such as camera dark noise) that are not considered in the simulation. Note that in our experiment, the coherence area of the speckle field is approximately 100 pixels, whereas in our model we assumed that each pixel experienced independent intensity fluctuations. However, we have performed extensive numerical simulations, such as those reported in Fig. 4, which show that the predictions 

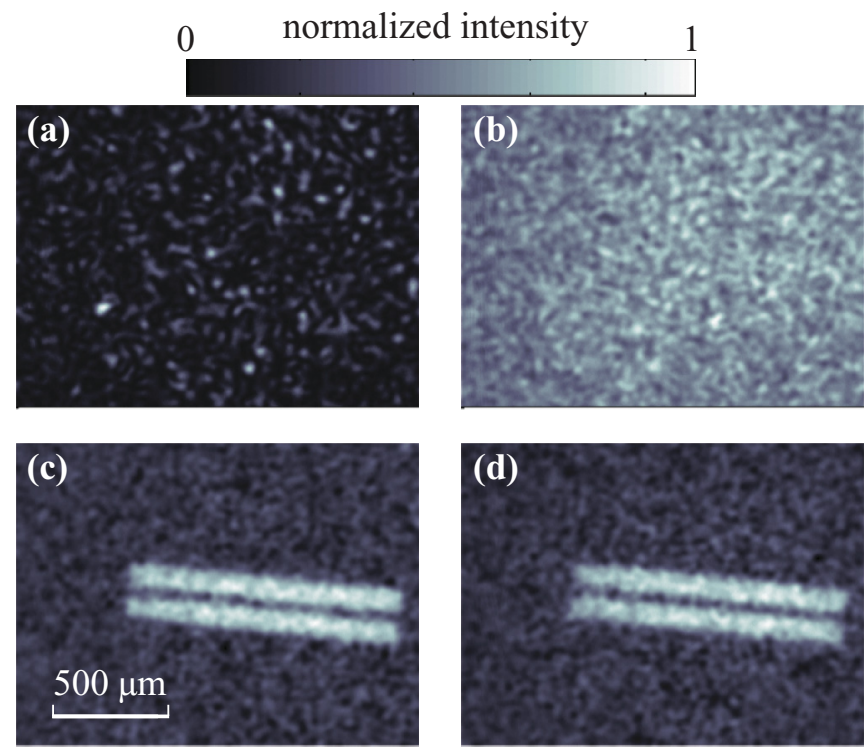

FIG. 3. (Color online) (a) Representative speckle pattern of the sort used in our experiments and (b) the intensity average of 25 patterns of the sort shown in (a). The statistics of the two patterns are very different, as described in the text. Nonetheless, ghost images obtained under the two conditions are essentially identical. (c) A ghost image of a double-bar pattern (1.2 mm long, $100 \mu \mathrm{m}$ wide, and with $40 \mu \mathrm{m}$ gap in between) taken using individual speckles and (d) a ghost image taken using the intensity average of $M=25$ individual speckle patterns. In each case, $N=10000$ measurements were used to obtain the ghost image.

of our model are not influenced by the average speckle size with respect the pixel size of the camera.

\section{SUMMARY}

In conclusion, we have presented a theoretical analysis with experimental demonstration which shows that the image quality of a thermal ghost imaging system is essentially independent of the response time of the detectors as compared

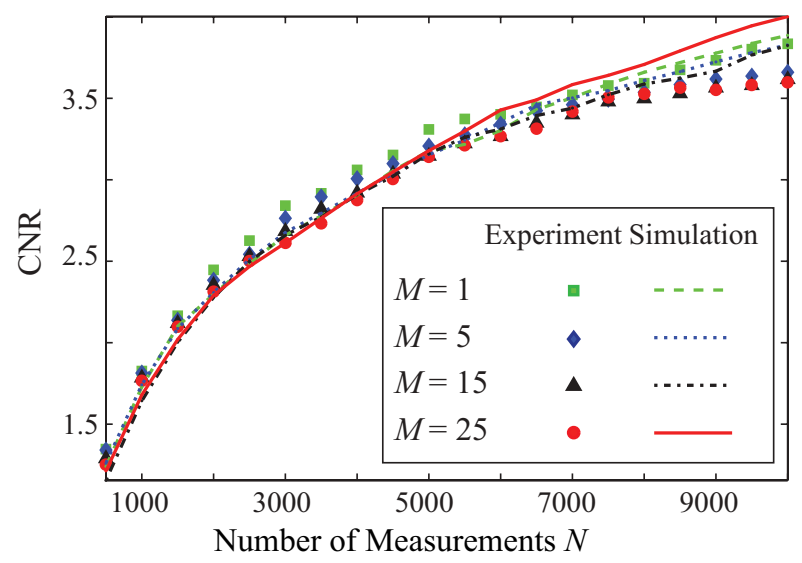

FIG. 4. (Color online) CNR as a function of the number of measurements $N$ for the ghost imaging system that responds to different numbers $M$ of averaged speckle patterns for each measurement. Here the symbols are experimental results, and the lines are simulation realizations. to the coherence time of the illuminating speckle fields. This surprising result arises from the fact that the image quality of a ghost imaging system is actually only weakly dependent on the kurtosis of the intensity fluctuation of the illuminating speckle field that the detectors respond to. As the detecting system becomes slow and sees only an average of multiple speckle patterns, the contrast ratio of the effective speckle field decreases monotonically, but the kurtosis actually converges to a value of 3 for thermal light. Consequently, the quality of the ghost image is almost not affected by the detector speed as long as all nonspeckle noise, such as the detector dark current noise, is small compared to the fluctuation of the averaged speckle fields.

While most thermal ghost imaging systems demonstrated to date have used pseudothermal light whose coherence time can be controlled to match the speed of the detectors, the possibility of performing ghost imaging with true thermal light has always been considered intriguing and highly desirable [16,26]. The work presented here shows that there need not be any blurring of the final image even when the detection system is much slower than coherence time of the thermal light source, as long as the illumination is strong enough that shot noise and detector noise can be neglected. Our result opens up the possibility of using slow detectors for thermal ghost imaging with quickly varying thermal speckle fields and may shed light on other applications using speckle fields as well.

\section{ACKNOWLEDGMENTS}

This work was supported by the DARPA/DSO InPho Program and by the Canada Excellence Research Chairs Program.

\section{APPENDIX: DERIVATION OF THE CNR OF THERMAL GHOST IMAGING}

Let $O(x)$ be the intensity transmission function of an object we wish to image. For simplicity we take the object to be binary, i.e., $O(x)=0$ or 1 . The object is also assumed to be pixelated and to transmit at a total of $T$ pixels. The object is illuminated with $N$ random intensity patterns, each represented by $I^{(n)}(x)$ for $n=1, \ldots, N$. For each illuminating pattern, the total energy transmitted through the object is recorded, denoted by $I_{\mathrm{B}}^{(n)}$. Given the assumption that the detected signal is primarily given by the random intensity variation of the speckle fields and that other noise sources, e.g., detector dark noise, can be neglected, the image can be acquired using the following correlation formula:

$$
G(x)=\frac{1}{N} \sum_{n=1}^{N} I^{(n)}(x) I_{\mathrm{B}}^{(n)}-\frac{1}{N^{2}} \sum_{n=1}^{N} I^{(n)}(x) \sum_{n=1}^{N} I_{\mathrm{B}}^{(n)} .
$$

We quantify the image quality by the CNR, which is given by the following relation:

$$
R_{\mathrm{CN}}=\frac{\left\langle G_{1}\right\rangle-\left\langle G_{0}\right\rangle}{\sqrt{\sigma_{1}^{2}+\sigma_{0}^{2}}}
$$

where $G_{1} \equiv G\left(x_{1}\right)$ for a point $x_{1}$ where $O\left(x_{1}\right)=1$ and $G_{0} \equiv$ $G\left(x_{0}\right)$ for a point $x_{0}$ where $O\left(x_{0}\right)=0$. Similarly, $\sigma_{1}^{2}$ and $\sigma_{0}^{2}$ are the variances of the signal at $x_{1}$ and $x_{0}$, respectively. 
We now derive in detail the expression of CNR in terms of the statistical properties of the illuminating light under a few simplifying, but reasonable, assumptions: (1) the intensity of the illuminating patterns are statistically independent from each other, and (2) the intensity of each pixel is independent of other pixels. Using the first assumption, we directly find the equations for the expected imaging signal,

and for the variance,

$$
\begin{aligned}
\sigma^{2}(x)= & \frac{1}{N}\left(\frac{N-1}{N}\right)^{2}\left\langle I^{2} I_{\mathrm{B}}^{2}\right\rangle+\frac{(N-1)(N-2)}{N^{3}}\left[\left\langle I^{2}\right\rangle\left\langle I_{\mathrm{B}}\right\rangle^{2}+\langle I\rangle^{2}\left\langle I_{\mathrm{B}}^{2}\right\rangle-\left\langle I I_{\mathrm{B}}\right\rangle^{2}\right]-2 \frac{1}{N}\left(\frac{N-1}{N}\right)^{2}\left[\left\langle I^{2} I_{\mathrm{B}}\right\rangle\left\langle I_{\mathrm{B}}\right\rangle+\left\langle I I_{\mathrm{B}}^{2}\right\rangle\langle I\rangle\right] \\
& +2 \frac{(N-1)(3 N-4)}{N^{3}}\left\langle I I_{\mathrm{B}}\right\rangle\langle I(x)\rangle\left\langle I_{\mathrm{B}}\right\rangle+\frac{N-1}{N^{3}}\left\langle I^{2}\right\rangle\left\langle I_{\mathrm{B}}^{2}\right\rangle-2 \frac{(2 N-3)(N-1)}{N^{3}}\langle I\rangle^{2}\left\langle I_{\mathrm{B}}\right\rangle^{2},
\end{aligned}
$$

where we have used the shorthand notation $I \equiv I(x)$.

Now, using the second assumption, we can further simplify the equations by substituting the following relations:

$$
\begin{gathered}
\langle I\rangle=\mu_{1}, \\
\left\langle I_{\mathrm{B}}\right\rangle=T \mu_{1}, \\
\left\langle I^{2}\right\rangle=\mu_{2}, \\
\left\langle I_{\mathrm{B}}^{2}\right\rangle=T(T-1) \mu_{1}^{2}+T \mu_{2}, \\
\left\langle I I_{\mathrm{B}}\right\rangle=(T-O(x)) \mu_{1}^{2}+O(x) \mu_{2}, \\
\left\langle I^{2} I_{\mathrm{B}}\right\rangle=\left(\mu_{3}-\mu_{2} \mu_{1}\right) O(x)+T \mu_{2} \mu_{1}, \\
\left\langle I I_{\mathrm{B}}^{2}\right\rangle=\left[\mu_{1}^{3}-\mu_{2} \mu_{1}+2 \mu_{1}(T-1)\left(\mu_{2}-\mu_{1}^{2}\right)\right] O(x)+T \mu_{2} \mu_{1}+T(T-1) \mu_{1}^{3}, \\
\left\langle I^{2} I_{\mathrm{B}}^{2}\right\rangle=\left[\mu_{4}-\mu_{2}^{2}+2 \mu_{1}(T-1)\left(\mu_{3}-\mu_{2} \mu_{1}\right)\right] O(x)+T \mu_{2}^{2}+T(T-1) \mu_{2} \mu_{1}^{2},
\end{gathered}
$$

where $\mu_{r} \equiv\left\langle I^{r}\right\rangle$ is the $r$ th moment of the intensity distribution of each illuminating pattern.

Using Eqs. (A3)-(A12), one can obtain the following expression for the numerator of Eq. (A2):

$$
\left\langle G_{1}\right\rangle-\left\langle G_{0}\right\rangle=\left(\frac{N-1}{N}\right) \sigma_{I}^{2},
$$

where we define $\sigma_{I}^{2} \equiv \mu_{2}-\mu_{1}^{2}$ as the variance of the intensities in each illuminating pattern. Similarly, we find the noise squared is given by

$$
\begin{aligned}
\sigma_{1}^{2}+\sigma_{0}^{2} & =\left(\frac{N-1}{N^{2}}\right)\left[2 T \sigma_{I}^{4}+(5-6 / N)\left(2 \mu_{2} \mu_{1}^{2}-\mu_{1}^{4}\right)-(2-3 / N) \mu_{2}^{2}-(1-1 / N)\left(4 \mu_{3} \mu_{1}-\mu_{4}\right)\right] \\
& =\left(\frac{N-1}{N^{2}}\right)\left[(2 T-2+3 / N) \sigma_{I}^{4}+(1-1 / N) \gamma_{I}^{4}\right],
\end{aligned}
$$

where $\gamma_{I}^{4} \equiv\left\langle\left(I-\mu_{1}\right)^{4}\right\rangle=\mu_{4}-4 \mu_{3} \mu_{1}+6 \mu_{2} \mu_{1}^{2}-3 \mu_{1}^{4}$ is the fourth-order moment about the mean of each illuminating pattern.

Substituting these relations in Eq. (A2), the CNR of the obtained ghost image is given by the remarkably simple formula

$$
R_{\mathrm{CN}}=\left[\frac{N-1}{(2 T-2+3 / N)+(1-1 / N)\left(\gamma_{I} / \sigma_{I}\right)^{4}}\right]^{1 / 2} .
$$

One sees that the CNR is determined by the number of measurements, the transmitting area relative to the spatial coherence area of the speckle field, and the quantity $\left(\gamma_{I} / \sigma_{I}\right)^{4}$, also known as the fourth standardized moment, or the kurtosis, of the intensity distribution of the illuminating speckle fields. 
[1] J. W. Goodman, Speckle Phenomena in Optics: Theory and Applications (Roberts, Englewood, CO, 2006).

[2] D. J. Pine, D. A. Weitz, P. M. Chaikin, and E. Herbolzheimer, Phys. Rev. Lett. 60, 1134 (1988).

[3] G. Montaldo, M. Tanter, and M. Fink, Phys. Rev. Lett. 106, 054301 (2011).

[4] D. A. Boas and A. K. Dunn, J Biomed. Opt. 15, 011109 (2010).

[5] C. Sanner, E. J. Su, A. Keshet, W. Huang, J. Gillen, R. Gommers, and W. Ketterle, Phys. Rev. Lett. 106, 010402 (2011).

[6] U. Bortolozzo, S. Residori, and P. Sebbah, Phys. Rev. Lett. 106, 103903 (2011).

[7] J. W. Goodman, J. Opt. Soc. Am. 66, 1145 (1976).

[8] T. B. Pittman, Y. H. Shih, D. V. Strekalov, and A. V. Sergienko, Phys. Rev. A 52, R3429 (1995).

[9] R. S. Bennink, S. J. Bentley, and R. W. Boyd, Phys. Rev. Lett. 89, 113601 (2002).

[10] A. Gatti, E. Brambilla, M. Bache, and L. A. Lugiato, Phys. Rev. Lett. 93, 093602 (2004).

[11] F. Ferri, D. Magatti, A. Gatti, M. Bache, E. Brambilla, and L. A. Lugiato, Phys. Rev. Lett. 94, 183602 (2005).

[12] A. Valencia, G. Scarcelli, M. D'Angelo, and Y. Shih, Phys. Rev. Lett. 94, 063601 (2005).

[13] Y.-H. Zhai, X.-H. Chen, D. Zhang, and L.-A. Wu, Phys. Rev. A 72, 043805 (2005).
[14] D. Zhang, Y.-H. Zhai, L.-A. Wu, and X.-H. Chen, Opt. Lett. 30, 2354 (2005).

[15] R. Meyers, K. S. Deacon, and Y. Shih, Phys. Rev. A 77, 041801(R) (2008).

[16] X.-H. Chen, Q. Liu, K.-H. Luo, and L.-A. Wu, Opt. Lett. 34, 695 (2009).

[17] G. Brida, M. V. Chekhova, G. A. Fornaro, M. Genovese, E. D. Lopaeva, and I. Ruo Berchera, Phys. Rev. A 83, 063807 (2011).

[18] G. Scarcelli, V. Berardi, and Y. Shih, Phys. Rev. Lett. 96, 063602 (2006).

[19] B. I. Erkmen and J. H. Shapiro, Phys. Rev. A 77, 043809 (2008).

[20] J. H. Shapiro and R. W. Boyd, Quantum Inf. Process. 11, 949 (2012).

[21] Y. Shih, Quantum Inf. Process. 11, 995 (2012).

[22] J. H. Shapiro and R. W. Boyd, Quantum Inf. Process. 11, 1003 (2012).

[23] Y. Bromberg, O. Katz, and Y. Silberberg, Phys. Rev. A 79, 053840 (2009).

[24] K. W. C. Chan, M. N. O'Sullivan, and R. W. Boyd, Opt. Exp. 18, 5562 (2010).

[25] K. W. C. Chan, M. N. O’Sullivan, and R. W. Boyd, Opt. Lett. 34, 3343 (2009).

[26] S. Karmakar, Y. Zhai, H. Chen, and Y. Shih (unpublished). 